\title{
Determinants of HPV-vaccination uptake and subgroups with a lower uptake in the Netherlands
}

\author{
A. C. de Munter ${ }^{1,2,5 \dagger}$, T. M. Schurink-van t Klooster ${ }^{4^{*} \dagger}$, A. van Lier ${ }^{4}$, R. Akkermans ${ }^{2,3}$, H. E. de Melker ${ }^{4}$ and \\ W. L. M. Ruijs ${ }^{1,4}$
}

\begin{abstract}
Background: In the Netherlands, the HPV-vaccine uptake was 52\% during the 2009 catch-up campaign (birth cohorts 1993-1996). This increased to 61\% in the regular immunization program (birth cohorts 2000-2001). However for birth cohorts 2003-2004 the uptake declined to 45.5\%. With this study we aimed to gain insight into social, economic and cultural determinants that are associated with HPV-vaccination uptake and which subgroups with a lower HPV-vaccination uptake can be identified. In addition, we investigated whether the influence of these factors changed over time.

Methods: To study the determinants of HPV-vaccine uptake we performed a database study using different aggregation levels, i.e. individual level, postal code level and municipality level. All Dutch girls who were invited for HPV-vaccination through the National Immunization Program in the years 2012, 2014 and 2017 (i.e. birth cohorts 1999, 2001 and 2004, respectively) were included in the study population. We conducted multilevel logistic regression analyses to analyze the influence of the determinants on HPV-vaccination uptake, taking into account that the delivery of HPV-vaccine was nested within municipalities.

Results: Results showed that in particular having not received a MMR-vaccination, having one or two parents born in Morocco or Turkey, living in an area with lower socioeconomic status and higher municipal voting proportions for Christian political parties or populist parties with liberal-conservative views were associated with a lower HPVvaccination uptake. Besides some changes in political preferences of the population and changes in the association between HPV uptake and urbanization level we found no clear determinants which could possibly explain the decrease in the HPV-vaccination uptake.
\end{abstract}

\footnotetext{
*Correspondence: tessa.schurink@rivm.nl

${ }^{\dagger}$ A. C. de Munter and T. M. Schurink-van t Klooster contributed equally to this work.

${ }^{4}$ Department National Immunization Program, Center of Epidemiology and Surveillance of Infectious Diseases, National Institute for Public Health and the Environment, PO box 1, 3720, BA, Bilthoven, The Netherlands Full list of author information is available at the end of the article
}

(c) The Author(s). 2021 Open Access This article is licensed under a Creative Commons Attribution 4.0 International License, which permits use, sharing, adaptation, distribution and reproduction in any medium or format, as long as you give appropriate credit to the original author(s) and the source, provide a link to the Creative Commons licence, and indicate if changes were made. The images or other third party material in this article are included in the article's Creative Commons licence, unless indicated otherwise in a credit line to the material. If material is not included in the article's Creative Commons licence and your intended use is not permitted by statutory regulation or exceeds the permitted use, you will need to obtain permission directly from the copyright holder. To view a copy of this licence, visit http://creativecommons.org/licenses/by/4.0/. The Creative Commons Public Domain Dedication waiver (http://creativecommons.org/publicdomain/zero/1.0/) applies to the data made available in this article, unless otherwise stated in a credit line to the data. 
Conclusions: In this study we identified current social, economic and cultural determinants that are associated with HPV-vaccination uptake and which low-vaccination subgroups can be identified. However, no clear determinants were found which could explain the decrease in the HPV-vaccination uptake. Tailored information and/or consultation for groups that are associated with a lower HPV-vaccination uptake might help to increase the HPVvaccination uptake in the future.

Keywords: Immunization, Human papillomavirus (HPV), Ethnicity, Urbanization, Socioeconomic status, Political preference

\section{Background}

Vaccination against human papillomavirus (HPV) targeting girls 12 years of age is part of the Dutch national immunization program (NIP) since 2010. Prior to this, a catch-up campaign for 13-16-year-old girls was initiated in 2009. The bivalent HPV16/18-vaccine was used starting with a three-dose schedule up to 2013 and a twodose schedule from 2014 onwards. HPV16 and - 18 together are estimated to account for $70 \%$ of all cases of cervical cancer [1]. In the Netherlands, annually about 800 women are diagnosed with cervical cancer and about 200 die due to this disease [2, 3].

The HPV-vaccination uptake is low compared to the coverage for other vaccines in the Dutch NIP. During the catch-up campaign in 2009, the vaccine coverage was 52\% for birth cohorts 1993-1996 [4]. This increased to $61 \%$ for birth cohorts 2000 and 2001 but declined thereafter to $45.5 \%$ for birth cohorts 2003 and 2004 [5]. In addition, large variations in the vaccination coverage were observed at municipality level ranging from less than $10 \%$ to more than $80 \%$ [6].

Research among girls who were targeted for the initial catch-up campaign and their mothers showed that socio-demographic determinants, such as socioeconomic status (SES) and country of birth were associated with HPV-vaccination uptake [7, 8]. In addition, various Christian groups have objections to HPV-vaccination because it concerns protection against a sexually transmitted infection or because they have religious objections to vaccination in general $[7,9,10]$. Previous studies indicate that in several high income countries lack of trust in the government also plays a role in the willingness to get HPV-vaccination [11-13]. An ecological study conducted in the United States showed that political color is associated with vaccination uptake in adolescence, as well [14]. In the Netherlands, high political preference for Protestant-Christian parties at municipality level was previously found to be associated with low HPV-vaccination uptake [7]. Political preference for other political parties might also be associated with low HPV-vaccination uptake, because of the relation with confidence in government institutions, media and social institutions $[15,16]$.

It is unknown whether the influence of the various social, economic and cultural determinants on HPVvaccination uptake changed over time in the Netherlands. In addition, it is unknown which determinants could explain the recent decrease in the HPVvaccination uptake. With this study, we aim to gain insight into the determinants that are associated with HPV-vaccination uptake and which low-vaccination subgroups can be identified, and to investigate whether target groups can be identified that are associated with the decline in HPV-vaccination uptake.

\section{Methods}

\section{Sample and data collection}

We performed a database study to investigate various determinants of HPV-vaccination uptake on different aggregation levels: individual, postal code and municipality. The sample included all girls invited for HPVvaccination through the NIP in the years 2012, 2014 and 2017, respectively from birth cohorts 1999, 2001 and 2004. For 2017 was the latest complete dataset available; in 2014 the vaccination schedule was changed and this was the last year before the decline in vaccination uptake; in 2012 and 2017 the Dutch National Elections for seats in the House of Representatives were held.

Anonymous individual-level data were obtained retrospectively in 2018 from the national vaccination register (Praeventis), using the 2018 municipality division (380 municipalities). The individual level variable Ethnicity was defined as country of birth of both parents, for which most common country of birth combinations were used.

Additional data, on postal code and municipality level, were extracted from the publicly available data of Statistics Netherlands (CBS), The Netherlands Institute for Social Research (SCP), and the Electoral Council (Kiesraad), or were provided by the Municipal Health Services (MHS). If data was not available for a certain invitation year, data of the most recent year was used (see Table 1 for variable details). 
Table 1 Characteristics of variables: level of aggregation, measurement level, year of data collection for each invitation year and original database

\begin{tabular}{|c|c|c|c|c|}
\hline Variable & Measurement level & $\begin{array}{l}\text { Invitation } \\
\text { Year }^{1}\end{array}$ & $\begin{array}{l}\text { Year of } \\
\text { data } \\
\text { collection }\end{array}$ & Database \\
\hline \multicolumn{5}{|l|}{ Individual-level } \\
\hline $\begin{array}{l}\text { HPV-vaccination status } \\
\text { (dependent variable) }\end{array}$ & $\begin{array}{l}\text { Dichotomous: } \\
\text { Completed series of HPV-vaccinations; } 0 \text { = has no completed HPV- } \\
\text { vaccination series; } 1 \text { = has a completed HPV-vaccination series (2012: 3- } \\
\text { doses; 2014/2017: 2-doses) }\end{array}$ & $\begin{array}{l}2012 \\
2014 \\
2017\end{array}$ & $\begin{array}{l}2018 \\
2018 \\
2018\end{array}$ & Praeventis \\
\hline MMR-vaccination status & $\begin{array}{l}\text { Categorical: } \\
\text { Zero, one, two doses of MMR-vaccination }\end{array}$ & $\begin{array}{l}2012 \\
2014 \\
2017\end{array}$ & $\begin{array}{l}2018 \\
2018 \\
2018\end{array}$ & Praeventis \\
\hline DT (aP)-IPV-vaccination status & $\begin{array}{l}\text { Categorical: } \\
\text { Zero, primary series (3-doses), completed series (6-doses) of DT (aP)-IPV- } \\
\text { vaccination }\end{array}$ & $\begin{array}{l}2012 \\
2014 \\
2017\end{array}$ & $\begin{array}{l}2018 \\
2018 \\
2018\end{array}$ & Praeventis \\
\hline Ethnicity $^{3}$ & $\begin{array}{l}\text { Categorical: } \\
14 \text { combinations of parents' country of birth }{ }^{4} \text { and the category unknown } \\
\text { (one or both parents' country of birth is unknown) }\end{array}$ & $\begin{array}{l}2012 \\
2014 \\
2017\end{array}$ & $\begin{array}{l}2018 \\
2018 \\
2018\end{array}$ & Praeventis \\
\hline \multicolumn{5}{|l|}{ Postal code-level } \\
\hline Socioeconomic status (SES) & $\begin{array}{l}\text { Categorical: } \\
\text { Status score low }(\leq-1.0000) \text {, } \\
\text { low-intermediate }(-0.9999 \text { to } 0.0000) \\
\text { high-intermediate }(0.0001-0.9999) \text {, high }(\geq 1.0000)\end{array}$ & $\begin{array}{l}2012 \\
2014 \\
2017\end{array}$ & $\begin{array}{l}2010 \\
2014 \\
2016\end{array}$ & SCP \\
\hline Road distance & $\begin{array}{l}\text { Categorical: } \\
0 \text { km (HPV-vaccination provided in same postal code as home address), } \\
0-5(0.1-4.9) \mathrm{km}, 5-10(5.0-9.9) \mathrm{km}, \geq 10 \mathrm{~km}\end{array}$ & $\begin{array}{l}2012 \\
2014 \\
2017\end{array}$ & $\begin{array}{l}2014 \\
2014 \\
2017\end{array}$ & MHS \\
\hline \multicolumn{5}{|l|}{ Municipality-level } \\
\hline Urbanization level $\left.\right|^{5}$ & $\begin{array}{l}\text { Categorical: } \\
\text { Very high }\left(>2500 \text { addresses per } \mathrm{km}^{2}\right) \text {, High }(1500-2500 \text { add. Per km²), } \\
\text { Moderately high }(1000-1500 \text { add. Per km²), Low }(500-1000 \text { add. Per km²), } \\
\text { Very low }\left(<500 \text { add. Per } \mathrm{km}^{2}\right)\end{array}$ & $\begin{array}{l}2012 \\
2014 \\
2017\end{array}$ & $\begin{array}{l}2017 \\
2017 \\
2017\end{array}$ & CBS \\
\hline $\begin{array}{l}\text { Voting proportions from the } \\
\text { National Elections for political } \\
\text { parties }^{6}\end{array}$ & $\begin{array}{l}\text { Dichotomous: } \\
\text { Voting proportion (percentage of votes per political party) lower or } \\
\text { higher than the mean of the national voting proportion of the party. }\end{array}$ & $\begin{array}{l}2012 \\
2014 \\
2017\end{array}$ & $\begin{array}{l}2012 \\
2012 \\
2017\end{array}$ & $\begin{array}{l}\text { Electoral } \\
\text { Council }\end{array}$ \\
\hline
\end{tabular}

Abbreviations: HPV Human Papillomavirus, MMR Mumps-measles-rubella, DTaP-IPV diphtheria-tetanus-pertussis-polio, SCP The Netherlands Institute for Social Research, MHS Municipal Health Services, CBS Statistics Netherlands, $k m$ kilometer. Praeventis National vaccination registry

${ }^{1}$ Girls invited for HPV-vaccination through the NIP in the years 2012, 2014 and 2017 were born in 1999, 2001 and 2004 respectively

2 If data was not available for a certain invitation year, data of the most recent year was used

${ }^{3}$ From December 2002 onwards, parents' country of birth was authorized from the Personal Records Database (Dutch: BRP, previously known as GBA) and therefore more complete for girls invited in 2017 (birth cohort 2004) than for girls invited in 2012 and 2014 (birth cohorts 1999 and 2001)

${ }^{4}$ The Netherlands-The Netherlands, The Netherlands-Turkey, Turkey-Turkey, The Netherlands-Morocco, Morocco-Morocco, The Netherlands-Surinam, SurinamSurinam, The Netherlands-Netherlands Antilles and Aruba, Netherlands Antilles and Aruba-Netherlands Antilles and Aruba, The Netherlands-other western country, other western country-other western country, The Netherlands-other non-western country, other non-western country -other non-western country, other western country-other non-western country, unknown

${ }^{5}$ In the database the urbanization level of 2017 was used; the most recent HPV-vaccination invitation year. Following the municipal re-division between 2017 and 2018, several municipalities merged into three new municipalities. For these three new municipalities we used the urbanization level of 2018

${ }^{6}$ Ten variables: 1) People's Party for Freedom and Democracy (VVD; right-wing liberal party with more progressive positions in ethical matters), 2) Labour Party (PvdA; progressive, social-democratic party) \& Denk (DENK; movement for migrants and a "tolerant and solidary society"; political party founded in 2015 by former members of the PvdA), 3) Party for Freedom (PVV; populist party with both conservative, liberal "right" and "left" views) \& Forum for Democracy (FvD; conservative, right-wing populist Eurosceptic political party; political party founded in 2015, whose voters are mainly former PVV voters), 4) Socialist Party (SP; socialist, Eurosceptic party which has a strong local, action-oriented basis), 5) Christian Democratic Appeal (CDA; Christian-inspired party at the center of the political spectrum), 6) Democrats 66 (D66; reformist social-liberal party), 7) Christian Union (CU; Christian party, with progressive positions in the social and ecological field and conservative positions on ethical issues) \& Reformed Political Party (SGP; conservative Christian (Reformed) party that wants to conduct politics strictly according to Biblical standards), 8) Green Left (GL; progressive party which attaches great importance to sustainability), 9) Party for the Animals (PvdD; testimonial party with main goals animal rights and animal welfare), 10) 50PLUS (50+; party that stands up especially for the interests of people aged 50 and over). The voting proportions for the three new municipalities in 2018 were calculated based on the weighted averages of the voting proportions of the previous municipalities before they were merged into the new municipality.

The postal code level variable Socioeconomic status was defined as status score, which is calculated by the SCP based on the educational level, paid jobs and income of households. Road distance was defined as distance by car between girls' home address and vaccination location in kilometers.

Voting proportions from the National Elections for political parties with 2 or more seats in the House were 
included in the analyses. Supplementary material 1 contains a list of these political parties and the distribution of seats in the House of Representatives in the Dutch National elections of 2012 and 2017 [17].

\section{Statistical analysis}

Multilevel logistic regression analyses were used to determine the association between the dependent variable HPV-vaccination uptake of a completed series ( 2 or 3 doses depending on invitation year) and predictor variables. The multilevel models included two hierarchical levels where girls who were invited for HPVvaccinations (level 1) were nested in municipalities (level 2). First, the associations between HPV-vaccination uptake and independent variables (Table 1) were measured using multilevel univariate logistic regression analyses [18]. Road distance to the vaccination location, SES and voting proportions for political parties were included on a categorical scale -instead of interval scale- to assess the relative effect of the predictor variables [19]. Secondly, multilevel multivariable logistic regression analysis was conducted. Predictor variables were selected based on a statistically significant association with HPV-vaccination uptake following the multilevel univariate logistic regression analysis $(p<0.05)$ unless multicollinearity $(>0.70)$ was found between two or more predictor variables. To calculate the correlation between all predictor variables in order to detect multicollinearity Spearman's correlation coefficient and the phi coefficient $(2 \times 2)$ were used [18]. In the multilevel multivariable logistic regression analysis, we used two different main models (Fig. 1). Model 1 contained a separate multilevel multivariable logistic regression model for each of the invitation years (2012, 2014 and 2017). In model 2, we combined the data of three invitation years using an additional variable for invitation year (categorial) and an interaction variable invitation year"predictor variable, to measure the effect of change of the predictor variables over time.

All analyses were performed using IBM SPSS Statistics ${ }^{\circ}$, version 24. Associations between HPV-vaccination uptake and predictor variables are shown with crude odds ratios (COR), adjusted odds ratios (AOR) and 95\% confidence intervals $(95 \% \mathrm{CI})$.

\section{Ethical considerations}

The study was approved by the research ethics committee of the Radboud University Nijmegen Medical Centre, Nijmegen, the Netherlands; CMO number 2018/4744.

\section{Results}

In the following paragraphs, main results of the multilevel univariate and multivariable logistic analysis of the invitation year 2017 are presented per predictor variable. Additionally, these results are compared to the associations between HPV-vaccination uptake and the predictor variables in the invitation years 2012 and 2014. Tables of the multilevel univariate analysis (Table 2) and multilevel multivariable logistic regression model (Table 3; model 1.3 for girls invited for HPV-vaccination in 2017) are included in this article. Model 1.1. and 1.2. (for the girls invited in 2012 and 2014) and the models including the interaction variable between invitation years and each predictor variable (models 2.1-2.14) can be found in supplementary material 2.

\section{MMR- and DT (aP)-IPV-vaccination status}

As the correlation between MMR-vaccination status and DT (aP)-IPV-vaccination status was $>0.80$ in the multicollinearity analysis, only MMR-vaccination status was included in the multilevel multivariable logistic regression models. In the multilevel univariate and multivariable models MMRvaccinations status was significant and positively associated

Model 1. Multilevel multivariable logistic regression anaysis per invitation year

Model 1.1 Model of invitation year 2012

Model 1.2 Model of invitation year 2014

Model 1.3 Model of invitation year 2017

Model 2. Multilevel multivariable logistic regression analysis invitation years 2012-2014-2017 including interaction variables

Model 2.1 Model of invitation years 2012-2014-2017, invitation year*MMR-vaccination status

Model 2.2 Model of invitation years 2012-2014-2017, invitation year* socioeconomic status

Model 2.3 Model of invitation years 2012-2014-2017, invitation year* road distance

Model 2.4 Model of invitation years 2012-2014-2017, invitation year*urbanization

Model 2.5-Model 2.14 Model of invitation years 2012-2014-2017, invitation year ${ }^{*}$ voting $\%$ of political parties in national elections

Fig. 1 Multilevel multivariable models used for statistical analysis 
Table 2 Descriptive analysis HPV-vaccination uptake and predictor variables on individual, postal code and municipality level $(n=$ 299,883)

\begin{tabular}{|c|c|c|c|c|c|c|c|c|c|c|c|c|}
\hline \multirow[b]{2}{*}{ Variable } & \multicolumn{4}{|l|}{2012} & \multicolumn{4}{|l|}{2014} & \multicolumn{4}{|l|}{2017} \\
\hline & $N$ & $\begin{array}{l}\text { HPV- } \\
\text { uptake } \\
\% \text { (a) }\end{array}$ & $\begin{array}{l}\text { Multi- } \\
\text { level } \\
\text { univariate } \\
\text { COR }\end{array}$ & $\begin{array}{l}p- \\
\text { value }\end{array}$ & $\mathbf{N}$ & $\begin{array}{l}\text { HPV- } \\
\text { uptake } \\
\%_{\text {(a) }}\end{array}$ & $\begin{array}{l}\text { Multi- } \\
\text { level } \\
\text { univariate } \\
\text { COR }\end{array}$ & $\begin{array}{l}\mathrm{p}- \\
\text { value }\end{array}$ & $\mathbf{N}$ & $\begin{array}{l}\text { HPV- } \\
\text { uptake } \\
\%^{\text {(a) }}\end{array}$ & $\begin{array}{l}\text { Multi- } \\
\text { level } \\
\text { univariate } \\
\text { COR }\end{array}$ & $\begin{array}{l}\mathrm{p}- \\
\text { value }\end{array}$ \\
\hline Total & 102,456 & $60.0 \%$ & & & 100,988 & $62.8 \%$ & & & 96,439 & $46.6 \%$ & & \\
\hline \multicolumn{13}{|l|}{ Individual level } \\
\hline MMR-vaccination status & 2012 & & & $<0.001$ & 2014 & & & $<0.001$ & 2017 & & & $<0.001$ \\
\hline Zero vaccinations & 5355 & $3.7 \%$ & reference & ref. & 3190 & $6.6 \%$ & ref. & ref. & 2548 & $6.0 \%$ & ref. & ref. \\
\hline One vaccination & 3079 & $28.4 \%$ & 9.72 & $<0.001$ & 3304 & $26.2 \%$ & 4.68 & $<0.001$ & 3162 & $13.3 \%$ & 2.24 & $<0.001$ \\
\hline Two Vaccinations & 94,022 & $64.3 \%$ & 44.05 & $<0.001$ & 94,494 & $66.0 \%$ & 25.34 & $<0.001$ & 90,729 & $48.9 \%$ & 13.95 & $<0.001$ \\
\hline $\begin{array}{l}\text { DT (aP)-IPV- } \\
\text { vaccination status }\end{array}$ & 2012 & & & $<0.001$ & 2014 & & & $<0.001$ & 2017 & & & $<0.001$ \\
\hline Zero vaccinations & 5549 & $6.1 \%$ & ref. & ref. & 3258 & $10.3 \%$ & ref. & ref. & 2656 & $8.2 \%$ & ref. & ref. \\
\hline Primary series & 2851 & $28.2 \%$ & 5.67 & $<0.001$ & 3088 & $25.9 \%$ & 2.81 & $<0.001$ & 3539 & $20.0 \%$ & 2.63 & $<0.001$ \\
\hline Completed series & 94,056 & $64.2 \%$ & 25.84 & $<0.001$ & 94,642 & $65.8 \%$ & 15.50 & $<0.001$ & 90,244 & $48.7 \%$ & 9.94 & $<0.001$ \\
\hline Ethnicity & 2012 & & & $<0.001$ & 2014 & & & $<0.001$ & 2017 & & & $<0.001$ \\
\hline NLD - NLD & 17,319 & $63.6 \%$ & ref. & ref. & 17,761 & $65.2 \%$ & ref. & ref. & 70,614 & $49.4 \%$ & ref. & ref. \\
\hline NLD - Turkey & 352 & $32.4 \%$ & 0.30 & $<0.001$ & 405 & $34.6 \%$ & 0.30 & $<0.001$ & 1023 & $26.0 \%$ & 0.34 & $<0.001$ \\
\hline Turkey - Turkey & 1306 & $27.9 \%$ & 0.26 & $<0.001$ & 1133 & $30.3 \%$ & 0.25 & $<0.001$ & 1802 & $20.5 \%$ & 0.24 & $<0.001$ \\
\hline NLD - Morocco & 199 & $36.2 \%$ & 0.36 & $<0.001$ & 224 & $35.7 \%$ & 0.34 & $<0.001$ & 762 & $18.8 \%$ & 0.22 & $<0.001$ \\
\hline Morocco - Morocco & 1272 & $18.2 \%$ & 0.16 & $<0.001$ & 1249 & $23.9 \%$ & 0.20 & $<0.001$ & 2920 & $16.5 \%$ & 0.18 & $<0.001$ \\
\hline NLD - Surinam & 369 & $52.8 \%$ & 0.73 & 0.003 & 414 & $54.8 \%$ & 0.70 & 0.001 & 927 & $41.3 \%$ & 0.71 & $<0.001$ \\
\hline Surinam - Surinam & 544 & $48.5 \%$ & 0.68 & $<0.001$ & 463 & $57.9 \%$ & 0.86 & 0.108 & 708 & $45.6 \%$ & 0.86 & 0.048 \\
\hline $\begin{array}{l}\text { NLD - Ned Antilles/ } \\
\text { Aruba }\end{array}$ & 186 & $51.6 \%$ & 0.65 & 0.004 & 161 & $57.8 \%$ & 0.74 & 0.058 & 475 & $35.6 \%$ & 0.55 & $<0.001$ \\
\hline $\begin{array}{l}\text { Ned Antilles/Aruba - } \\
\text { Ned Antilles/Aruba }\end{array}$ & 214 & $23.8 \%$ & 0.20 & $<0.001$ & 150 & $44.7 \%$ & 0.45 & $<0.001$ & 183 & $35.5 \%$ & 0.57 & $<0.001$ \\
\hline NLD - other WC & 748 & $58.6 \%$ & 0.84 & 0.020 & 858 & $64.1 \%$ & 0.95 & 0.493 & 2331 & $51.0 \%$ & 1.03 & 0.573 \\
\hline other WC - other WC & 430 & $43.5 \%$ & 0.44 & $<0.001$ & 468 & $53.2 \%$ & 0.62 & $<0.001$ & 704 & $46.9 \%$ & 0.84 & 0.025 \\
\hline NLD - other NWC & 490 & $58.0 \%$ & 0.84 & 0.071 & 581 & $59.9 \%$ & 0.82 & 0.027 & 1673 & $50.0 \%$ & 0.99 & 0.778 \\
\hline $\begin{array}{l}\text { other NWC - other } \\
\text { NWC }\end{array}$ & 1184 & $47.6 \%$ & 0.57 & $<0.001$ & 1192 & $59.3 \%$ & 0.83 & 0.004 & 2190 & $51.1 \%$ & 1.05 & 0.252 \\
\hline other WC - other NWC & 92 & $43.5 \%$ & 0.50 & 0.001 & 104 & $44.2 \%$ & 0.45 & $<0.001$ & 196 & $35.7 \%$ & 0.53 & $<0.001$ \\
\hline Unknown & 77,751 & $61.2 \%$ & 0.85 & $<0.001$ & 75,825 & $63.9 \%$ & 0.94 & 0.003 & 9931 & $42.9 \%$ & 0.74 & $<0.001$ \\
\hline \multicolumn{13}{|l|}{ Postal code level } \\
\hline $\begin{array}{l}\text { Socioeconomic status } \\
\text { (b) }\end{array}$ & 2012 & & & $<0.001$ & 2014 & & & $<0.001$ & $2017^{*}$ & & & $<0.001$ \\
\hline Low & 17,723 & $51.4 \%$ & ref. & ref. & 19,395 & $54.9 \%$ & ref. & ref. & 18,149 & $36.9 \%$ & ref. & ref. \\
\hline Low-intermediate & 29,714 & $58.8 \%$ & 1.27 & $<0.001$ & 26,556 & $62.9 \%$ & 1.34 & $<0.001$ & 25,467 & $44.6 \%$ & 1.36 & $<0.001$ \\
\hline High-intermediate & 38,297 & $62.7 \%$ & 1.50 & $<0.001$ & 37,747 & $64.4 \%$ & 1.57 & $<0.001$ & 34,292 & $48.8 \%$ & 1.64 & $<0.001$ \\
\hline High & 15,981 & $65.3 \%$ & 1.84 & $<0.001$ & 16,732 & $68.4 \%$ & 1.93 & $<0.001$ & 18,099 & $54.8 \%$ & 2.09 & $<0.001$ \\
\hline Road distance & $2012^{*}$ & & & 0.129 & 2014 & & & 0.031 & 2017 & & & 0.340 \\
\hline $0 \mathrm{~km}$ & 12,694 & $60.3 \%$ & ref. & ref. & 13,661 & $62.3 \%$ & ref. & ref. & 13,104 & $45.8 \%$ & ref. & ref. \\
\hline $0-5 \mathrm{~km}$ & 44,246 & $59.3 \%$ & 1.03 & 0.279 & 41,395 & $62.0 \%$ & 1.04 & 0.105 & 38,045 & $46.9 \%$ & 1.02 & 0.547 \\
\hline $5-10 \mathrm{~km}$ & 28,986 & $60.6 \%$ & 1.06 & 0.026 & 28,560 & $63.9 \%$ & 1.07 & 0.003 & 29,079 & $46.7 \%$ & 1.04 & 0.081 \\
\hline$>10 \mathrm{~km}$ & 16,495 & $60.8 \%$ & 1.05 & 0.115 & 17,343 & $63.3 \%$ & 1.05 & 0.072 & 16,185 & $46.2 \%$ & 1.02 & 0.333 \\
\hline
\end{tabular}


Table 2 Descriptive analysis HPV-vaccination uptake and predictor variables on individual, postal code and municipality level $(n=$ 299,883) (Continued)

\begin{tabular}{|c|c|c|c|c|c|c|c|c|c|c|c|c|}
\hline \multirow[b]{2}{*}{ Variable } & \multicolumn{4}{|l|}{2012} & \multicolumn{4}{|l|}{2014} & \multicolumn{4}{|l|}{2017} \\
\hline & $\bar{N}$ & $\begin{array}{l}\text { HPV- } \\
\text { uptake } \\
\% \text { (a) }\end{array}$ & $\begin{array}{l}\text { Multi- } \\
\text { level } \\
\text { univariate } \\
\text { COR }\end{array}$ & $\begin{array}{l}p \text { - } \\
\text { value }\end{array}$ & $\bar{N}$ & $\begin{array}{l}\text { HPV- } \\
\text { uptake } \\
\%^{\text {(a) }}\end{array}$ & $\begin{array}{l}\text { Multi- } \\
\text { level } \\
\text { univariate } \\
\text { COR }\end{array}$ & $\begin{array}{l}\mathrm{p}- \\
\text { value }\end{array}$ & $\bar{N}$ & $\begin{array}{l}\text { HPV- } \\
\text { uptake } \\
\% \text { (a) }\end{array}$ & $\begin{array}{l}\text { Multi- } \\
\text { level } \\
\text { univariate } \\
\text { COR }\end{array}$ & $\begin{array}{l}\mathrm{p}- \\
\text { value }\end{array}$ \\
\hline \multicolumn{13}{|l|}{ Municipality level } \\
\hline Urbanization level ${ }^{(c)}$ & $2012^{*}$ & & & 0.020 & $2014 *$ & & & 0.134 & 2017 & & & 0.058 \\
\hline Very high & 23,398 & $53.3 \%$ & ref. & ref. & 19,446 & $57.9 \%$ & ref. & ref. & 19,280 & $45.3 \%$ & ref. & ref. \\
\hline High & 31,870 & $60.3 \%$ & 1.14 & 0.272 & 30,512 & $62.9 \%$ & 1.03 & 0.793 & 29,502 & $46.1 \%$ & 0.95 & 0.642 \\
\hline Moderately high & 17,260 & $62.7 \%$ & 1.28 & 0.038 & 18,314 & $64.0 \%$ & 1.16 & 0.232 & 17,512 & $47.9 \%$ & 1.03 & 0.819 \\
\hline Low & 21,315 & $63.8 \%$ & 1.36 & 0.007 & 23,200 & $65.5 \%$ & 1.22 & 0.099 & 21,526 & $48.3 \%$ & 1.07 & 0.572 \\
\hline Very low & 8604 & $63.1 \%$ & 1.25 & 0.062 & 9513 & $64.1 \%$ & 1.11 & 0.390 & 8618 & $44.3 \%$ & 0.87 & 0.266 \\
\hline $\begin{array}{l}\text { Voting } \% \text { political } \\
\text { parties }(d)\end{array}$ & 2012 & & & & $2014^{*}$ & & & & 2017 & & & \\
\hline \multicolumn{13}{|c|}{ Lower or higher than national mean } \\
\hline \multicolumn{13}{|c|}{ People's Party for Freedom and Democracy (VVD) } \\
\hline Lower & 64,595 & $57.3 \%$ & ref. & ref. & 60,833 & $60.5 \%$ & ref. & ref. & 58,855 & $43.6 \%$ & ref. & ref. \\
\hline Higher & 37,852 & $64.8 \%$ & 1.30 & $<0.001$ & 40,152 & $66.3 \%$ & 1.27 & $<0.001$ & 37,583 & $51.3 \%$ & 1.47 & $<0.001$ \\
\hline \multicolumn{13}{|c|}{ Labor Party (PvdA), Denk (DENK) } \\
\hline Lower & 36,543 & $60.9 \%$ & reference & ref. & 39,038 & $62.7 \%$ & ref. & ref. & 34,880 & $46.7 \%$ & ref. & ref. \\
\hline Higher & 65,904 & $59.6 \%$ & 1.09 & 0.096 & 61,947 & $62.9 \%$ & 1.10 & 0.076 & 61,558 & $46.5 \%$ & 1.00 & 0.931 \\
\hline \multicolumn{13}{|c|}{ Party for Freedom (PPV), Forum for Democracy (FvD) } \\
\hline Lower & 57,814 & $59.7 \%$ & reference & ref. & 56,784 & $62.3 \%$ & ref. & ref. & 52,307 & $48.1 \%$ & ref. & ref. \\
\hline Higher & 44,633 & $60.5 \%$ & 1.14 & 0.007 & 44,201 & $63.5 \%$ & 1.10 & 0.077 & 44,131 & $44.8 \%$ & 0.88 & 0.006 \\
\hline \multicolumn{13}{|l|}{ Socialist Party (SP) } \\
\hline Lower & 52,335 & $57.6 \%$ & reference & ref. & 53,199 & $59.8 \%$ & ref. & ref. & 4665 & $44.3 \%$ & ref. & ref. \\
\hline Higher & 50,112 & $62.6 \%$ & 1.40 & $<0.001$ & 47,786 & $66.2 \%$ & 1.45 & $<0.001$ & 41,773 & $49.5 \%$ & 1.29 & $<0.001$ \\
\hline \multicolumn{13}{|c|}{ Christian Democratic Appeal (CDA) } \\
\hline Lower & 77,344 & $59.9 \%$ & ref. & ref. & 73,747 & $63.0 \%$ & ref. & ref. & 71,075 & $46.8 \%$ & ref. & ref. \\
\hline Higher & 25,103 & $60.6 \%$ & 0.94 & 0.201 & 27,238 & $62.4 \%$ & 0.97 & 0.487 & 25,363 & $45.9 \%$ & 0.94 & 0.221 \\
\hline \multicolumn{13}{|l|}{ Democrats 66 (D66) } \\
\hline Lower & 43,514 & $59.9 \%$ & ref. & ref. & 45,849 & $61.2 \%$ & ref. & ref. & 38,093 & $42.7 \%$ & ref. & ref. \\
\hline Higher & 58,933 & $60.1 \%$ & 1.25 & $<0.001$ & 55,136 & $64.2 \%$ & 1.30 & $<0.001$ & 58,345 & $49.1 \%$ & 1.46 & $<0.001$ \\
\hline \multicolumn{13}{|c|}{ Christian Union (CU), Reformed Political Party (SGP) } \\
\hline Lower & 78,482 & $61.8 \%$ & ref. & ref. & 75,461 & $64.9 \%$ & ref. & ref. & 71,521 & $48.5 \%$ & ref. & ref. \\
\hline Higher & 23,965 & $54.3 \%$ & 0.59 & $<0.001$ & 25,524 & $56.7 \%$ & 0.60 & $<0.001$ & 24,917 & $41.1 \%$ & 0.62 & $<0.001$ \\
\hline \multicolumn{13}{|l|}{ Green Left (GL) } \\
\hline Lower & 44,692 & $62.0 \%$ & ref. & ref. & 47,846 & $63.2 \%$ & ref. & ref. & 38,670 & $45.0 \%$ & ref. & ref. \\
\hline Higher & 57,755 & $58.6 \%$ & 1.07 & 0.196 & 53,139 & $62.5 \%$ & 1.11 & 0.046 & 57,768 & $47.6 \%$ & 1.21 & $<0.001$ \\
\hline \multicolumn{13}{|c|}{ Party for the Animals (PvdD) } \\
\hline Lower & 37,964 & $61.9 \%$ & ref. & ref. & 40,832 & $63.7 \%$ & ref. & ref. & 38,633 & $46.7 \%$ & ref. & ref. \\
\hline Higher & 64,483 & $59.0 \%$ & 1.03 & 0.561 & 60,153 & $62.2 \%$ & 1.02 & 0.737 & 57,805 & $46.5 \%$ & 1.03 & 0.588 \\
\hline
\end{tabular}


Table 2 Descriptive analysis HPV-vaccination uptake and predictor variables on individual, postal code and municipality level $(n=$ 299,883) (Continued)

\begin{tabular}{|c|c|c|c|c|c|c|c|c|c|c|c|c|}
\hline \multirow[b]{2}{*}{ Variable } & \multicolumn{4}{|l|}{2012} & \multicolumn{4}{|l|}{2014} & \multicolumn{4}{|l|}{2017} \\
\hline & $\bar{N}$ & $\begin{array}{l}\text { HPV- } \\
\text { uptake } \\
\% \text { (a) }\end{array}$ & $\begin{array}{l}\text { Multi- } \\
\text { level } \\
\text { univariate } \\
\text { COR }\end{array}$ & $\begin{array}{l}p- \\
\text { value }\end{array}$ & $\bar{N}$ & $\begin{array}{l}\text { HPV- } \\
\text { uptake } \\
\%_{\text {(a) }}\end{array}$ & $\begin{array}{l}\text { Multi- } \\
\text { level } \\
\text { univariate } \\
\text { COR }\end{array}$ & $\begin{array}{l}\mathrm{p}- \\
\text { value }\end{array}$ & $\bar{N}$ & $\begin{array}{l}\text { HPV- } \\
\text { uptake } \\
\%_{\text {(a) }}\end{array}$ & $\begin{array}{l}\text { Multi- } \\
\text { level } \\
\text { univariate } \\
\text { COR }\end{array}$ & $\begin{array}{l}\mathrm{p}- \\
\text { value }\end{array}$ \\
\hline \multicolumn{13}{|c|}{ 50PLUS (50+) } \\
\hline Lower & 57,554 & $58.1 \%$ & reference & ref. & 55,729 & $61.0 \%$ & ref. & ref. & 55,484 & $46.5 \%$ & ref. & ref. \\
\hline Higher & 44,893 & $62.6 \%$ & 1.31 & $<0.001$ & 45,256 & $65.0 \%$ & 1.25 & $<0.001$ & 40,954 & $46.7 \%$ & 1.10 & 0.052 \\
\hline
\end{tabular}

Abbreviations: COR crude odds ratio, MMR mumps-measles-rubella, DT (aP)-IPV diphtheria-tetanus-pertussis-polio, NL the Netherlands, Ned Antilles/ Aruba the Netherlands Antilles and Aruba, WC western countries, NWC non-western countries. km kilometer, VVD People's Party for Freedom and Democracy, PvdA Labor Party, PVV Party for Freedom, FvD Forum for Democracy, SP Socialist Party, CDA Christian Democratic Appeal, D66 Democrats 66, CU Christian Union, SGP Reformed Political Party, GL Green Left, PvdD The Party for the Animals, 50 + =50PLUS. For explanatory notes on the political parties we refer to Supplementary material 1

(a) HPV-uptake $\%=\%$ of total of girls (N) with a completed HPV-vaccination series. Girls invited in 2012 were offered a three-dose series, girls invited in 2014 and 2017 a 2-dose series.

(b) Socioeconomic status classification; low ( $\leq-1.0000)$, low-intermediate $(-0.9999$ to 0.0000$)$, high-intermediate (0.0001-0.9999), high ( $\geq 1.0000)$. (c) Urbanization classification; Very high: > 2500 addresses per km2, high: 1500-2500 addresses per km2, moderately high: $1000-1500$ addresses per km2, low: 500-1000 addresses per km2, very low < 500 addresses per km2.

(d) Voting \% classification: higher or lower compared to the national mean.

* For this variable/ invitation year, data from the most recent year available was used (See Table 1 for variable details)

with HPV-vaccination uptake (Tables 2, 3 and supplementary material 2 - model 1.1, 1.2), indicating that girls who did not have a completed series of MMR-vaccination had a lower HPV-vaccination uptake.

\section{Ethnicity}

Overall, girls with one or two parents born in another country than the Netherlands (both western and nonwestern countries) had a significantly lower HPVvaccination uptake compared to girls whose parents both were born in the Netherlands (Tables 2, 3 and supplementary material 2 - model 1.1, 1.2).

In each invitation year girls with one or two parents born in Morocco or Turkey showed a significantly lower HPV-vaccination uptake compared to girls with two parents born in the Netherlands (Table 3 and supplementary material 2 - model 1.1, 1.2).

Considering the high number of girls of whom the country of birth of one or two parents is unknown in 2012 and 2014, compared to less unknown values in 2017, the effect of change over time on ethnicity could not be compared in a multilevel multivariate logistic regression model.

\section{Socioeconomic status (SES)}

Girls who lived in lower SES postal code areas had a statistically significant lower HPV-vaccination uptake than girls who lived in higher SES postal code areas (Tables 2, 3 , and supplementary material 2 - model 1.1, 1.2). In each invitation year the odds of having received a completed series of HPV-vaccination was highest among girls who lived in a high SES postal code area compared to girls who lived in a low SES postal code area, followed by girls who lived in a high-intermediate SES postal code area, and subsequently, girls who lived in a low- intermediate SES postal code area (Tables 2, 3 and supplementary material 2 - model 1.1, 1.2).

\section{Road distance}

In 2017, the multilevel univariate logistic regression model indicated no statistical significant difference HPVvaccination uptake among girls who lived closer or further away from the vaccination location (Table 2). However, the multivariable models showed that girls who lived in a postal code area which was five or more kilometers from the postal code area of the vaccination location, had a very small but statistically significant lower odds of having received a completed series of HPV-vaccinations compared to girls living in the same postal code area as the vaccination location (Table 3 and supplementary material 2 model 2.3)., This association was not significant in the multilevel multivariable models of 2012 and 2014 (supplementary material 2 - model 1.1, 1.2).

\section{Urbanization level}

In the multilevel univariate logistic regression model no statistically significant association was found between municipal urbanization level and girls' HPV-vaccination uptake in 2017 (Table 2). In the multivariable logistic regression analysis (Table 3), girls who were invited for HPV-vaccination in 2017 and lived in a municipality with a high or moderately high urbanization level had a statistically significant lower HPV-vaccination uptake compared to girls who lived in a very high urban municipality. The multilevel multivariable logistic regression models of invitation year 2012 and 2014 showed that girls living in low and very low urban municipalities had a statistically significant higher HPV-vaccination uptake than girls living in very high urban municipalities (supplementary material 2 
Table 3 Multilevel multivariable logistic regression analysis of invitation year 2017, model 1.3, ( $n=96,007 ; 99.6 \%$ of the girls included in model)

\begin{tabular}{|c|c|c|c|c|c|}
\hline Variable & $\mathrm{N}$ & $\begin{array}{l}\text { HPV-uptake } \\
\%^{\text {(a) }}\end{array}$ & Adjusted OR (AOR) & $95 \% \mathrm{Cl}$ & p-value \\
\hline MMR-vaccination status & & & & & $<0.001$ \\
\hline Zero vaccinations & 2541 & $6.0 \%$ & reference & ref. & ref. \\
\hline One vaccination & 3155 & $13.3 \%$ & 2.38 & $1.96-2.89$ & $<0.001$ \\
\hline Two Vaccinations & 90,311 & $48.9 \%$ & 14.69 & $12.44-17.35$ & $<0.001$ \\
\hline Ethnicity & & & & & $<0.001$ \\
\hline$N L-N L$ & 70,228 & $49.4 \%$ & ref. & ref. & ref. \\
\hline NL - Turkey & 1021 & $26.1 \%$ & 0.37 & $0.32-0.42$ & $<0.001$ \\
\hline Turkey - Turkey & 1800 & $20.5 \%$ & 0.27 & $0.24-0.31$ & $<0.001$ \\
\hline $\mathrm{NL}$ - Morocco & 760 & $18.7 \%$ & 0.23 & $0.19-0.28$ & $<0.001$ \\
\hline Morocco - Morocco & 2920 & $16.5 \%$ & 0.20 & $0.18-0.23$ & $<0.001$ \\
\hline NL- Surinam & 924 & $41.5 \%$ & 0.75 & $0.65-0.86$ & $<0.001$ \\
\hline Surinam - Surinam & 707 & $45.7 \%$ & 0.94 & $0.81-1.10$ & 0.451 \\
\hline NL - Ned Antilles/Aruba & 475 & $35.6 \%$ & 0.60 & $0.49-0.73$ & $<0.001$ \\
\hline Ned Antilles/Aruba - Ned Antilles/Aruba & 182 & $35.7 \%$ & 0.83 & $0.60-1.15$ & 0.266 \\
\hline NL - other WC & 2320 & $51.0 \%$ & 1.07 & $0.98-1.16$ & 0.142 \\
\hline other WC - other WC & 704 & $46.9 \%$ & 1.17 & $0.99-1.37$ & 0.065 \\
\hline NL - other NWC & 1667 & $50.0 \%$ & 1.03 & $0.93-1.14$ & 0.555 \\
\hline other NWC - other NWC & 2188 & $51.1 \%$ & 1.25 & $1.14-1.37$ & $<0.001$ \\
\hline other WC - other NWC & 196 & $35.7 \%$ & 0.65 & $0.48-0.88$ & 0.005 \\
\hline Unknown & 9915 & $42.9 \%$ & 0.91 & $0.87-0.95$ & $<0.001$ \\
\hline Socioeconomic status ${ }^{(b)}$ & & & & & $<0.001$ \\
\hline Low & 18,149 & $36.9 \%$ & ref. & ref. & ref. \\
\hline Low - intermediate & 25,467 & $44.6 \%$ & 1.21 & $1.15-1.27$ & $<0.001$ \\
\hline High - intermediate & 34,292 & $48.8 \%$ & 1.40 & $1.34-1.47$ & $<0.001$ \\
\hline High & 18,099 & $54.8 \%$ & 1.68 & $1.59-1.77$ & $<0.001$ \\
\hline Road distance & & & & & $<0.001$ \\
\hline $0 \mathrm{~km}$ & 13,104 & $45.8 \%$ & ref. & ref. & ref. \\
\hline $0-5 \mathrm{~km}$ & 37,943 & $46.9 \%$ & 0.99 & $0.94-1.03$ & 0.555 \\
\hline $5-10 \mathrm{~km}$ & 28,925 & $46.7 \%$ & 0.93 & $0.89-0.98$ & 0.006 \\
\hline$>10 \mathrm{~km}$ & 16,035 & $46.2 \%$ & 0.90 & $0.85-0.95$ & $<0.001$ \\
\hline Urbanization level ${ }^{(c)}$ & & & & & 0.002 \\
\hline Very high & 19,258 & $45.3 \%$ & ref. & ref. & ref. \\
\hline High & 29,441 & $46.1 \%$ & 0.84 & $0.70-0.995$ & 0.043 \\
\hline Moderately high & 17,432 & $47.9 \%$ & 0.75 & $0.62-0.90$ & 0.002 \\
\hline Low & 21,360 & $48.3 \%$ & 0.89 & $0.74-1.08$ & 0.244 \\
\hline Very low & 8616 & $44.1 \%$ & 0.86 & $0.70-1.05$ & 0.131 \\
\hline \multicolumn{6}{|c|}{ Voting \% People's Party for Freedom and Democracy (VVD) } \\
\hline Lower & 58,531 & $43.5 \%$ & ref. & ref. & ref. \\
\hline Higher & 37,476 & $51.3 \%$ & 1.22 & $1.12-1.33$ & $<0.001$ \\
\hline \multicolumn{6}{|l|}{ Voting \% Labor Party (PvdA), Denk (DENK) } \\
\hline Lower & 34,742 & $46.8 \%$ & ref. & ref. & ref. \\
\hline Higher & 61,265 & $46.5 \%$ & 0.94 & $0.86-1.04$ & 0.209 \\
\hline
\end{tabular}


Table 3 Multilevel multivariable logistic regression analysis of invitation year 2017, model 1.3, $(n=96,007 ; 99.6 \%$ of the girls included in model) (Continued)

\begin{tabular}{|c|c|c|c|c|c|}
\hline Variable & $\mathrm{N}$ & $\begin{array}{l}\text { HPV-uptake } \\
\% \text { (a) }\end{array}$ & Adjusted OR (AOR) & $95 \% \mathrm{Cl}$ & p-value \\
\hline \multicolumn{6}{|c|}{ Voting \% Party for Freedom (PVV), Forum for Democracy (FvD) } \\
\hline Lower & 52,033 & $48.1 \%$ & ref. & ref. & ref. \\
\hline Higher & 43,974 & $44.8 \%$ & 0.90 & $0.81-0.99$ & 0.029 \\
\hline \multicolumn{6}{|c|}{ Voting \% Socialist Party (SP) } \\
\hline Lower & 54,491 & $44.3 \%$ & ref. & ref. & ref. \\
\hline Higher & 41,516 & $49.5 \%$ & 1.39 & $1.27-1.53$ & $<0.001$ \\
\hline \multicolumn{6}{|c|}{ Voting \% Christian Democratic Appeal (CDA) } \\
\hline Lower & 70,868 & $46.8 \%$ & ref. & ref. & ref. \\
\hline Higher & 25,139 & $45.9 \%$ & 0.89 & $0.80-0.99$ & 0.026 \\
\hline \multicolumn{6}{|c|}{ Voting \% Democrats 66 (D66) } \\
\hline Lower & 37,814 & $42.6 \%$ & ref. & ref. & ref. \\
\hline Higher & 58,193 & $49.1 \%$ & 1.17 & $1.05-1.30$ & 0.003 \\
\hline \multicolumn{6}{|c|}{ Voting \% Christian Union (CU), Reformed Political Party (SGP) } \\
\hline Lower & 71,226 & $48.5 \%$ & ref. & ref. & ref. \\
\hline Higher & 24,781 & $41.1 \%$ & 0.81 & $0.73-0.91$ & $<0.001$ \\
\hline \multicolumn{6}{|c|}{ Voting \% Green Left (GL) } \\
\hline Lower & 38,470 & $45.0 \%$ & ref. & ref. & ref. \\
\hline Higher & 57,537 & $47.6 \%$ & 1.15 & $1.03-1.30$ & 0.015 \\
\hline \multicolumn{6}{|c|}{ Voting \% Party for the Animals (PvdD) } \\
\hline Lower & 38,403 & $46.7 \%$ & ref. & ref. & ref. \\
\hline Higher & 57,604 & $46.5 \%$ & 0.82 & $0.74-0.91$ & $<0.001$ \\
\hline \multicolumn{6}{|c|}{ Voting \% 50PLUS (50+) } \\
\hline Lower & 55,213 & $46.5 \%$ & ref. & ref. & ref. \\
\hline Higher & 40,794 & $46.6 \%$ & 0.99 & $0.90-1.09$ & 0.814 \\
\hline
\end{tabular}

Abbreviations: OR odds ratio, $C l$ confidence interval, MMR mumps-measles-rubella, NL the Netherlands, Ned Antilles/Aruba the Netherlands Antilles and Aruba, WC western countries, NWC non-western countries, km kilometer, VVD People's Party for Freedom and Democracy, PvdA Labor Party, PVV Party for Freedom, FvD Forum for Democracy, SP Socialist Party, CDA Christian Democratic Appeal, D66 Democrats 66, CU Christian Union, SGP Reformed Political Party, GL Green Left, $P v d D$ The Party for the Animals, $50+=50$ PLUS. For explanatory notes on the political parties we refer to Supplementary material 1

(a) HPV-uptake $\%=\%$ of total of girls (N) with a completed HPV-vaccination series (2 doses).

(b) Socioeconomic status classification; low $(\leq-1.0000)$, low-intermediate $(-0.9999$ to 0.0000$)$, high-intermediate (0.0001-0.9999), high ( $\geq 1.0000)$.

(c) Urbanization classification; Very high: $>2500$ addresses per km2, high: 1500-2500 addresses per km2, moderately high: $1000-1500$ addresses per km2, low:

500-100 addresses per $\mathrm{km} 2$, very low $<500$ addresses per $\mathrm{km} 2$

(d) Voting \% classification: higher or lower compared to the national mean.

- model 1.1, 1.2). In the multilevel multivariable logistic regression analysis including the interaction variable invitation year"urbanization level, no statistically significant different effect was found for urbanization level between the invitation years 2012 and 2014. However, in invitation year 2017, the effect of urbanization is statistically significant different from invitation year 2012, i.e. the difference in HPV-vaccination uptake between different levels of urbanization becomes smaller (supplementary material 2 - model 2.4).

\section{Voting proportions of political parties in national elections}

The multilevel univariate and multivariable logistic regression analysis of 2017 showed a positive association between HPV-vaccination uptake and municipal voting proportion for People's Party for Freedom and Democracy (VVD), Socialist Party (SP), Democrats 66 (D66) and Green Left (GL) (Tables 2 and 3). This indicates that girls who lived in a municipality with a higher voting proportion for these parties, compared to the national mean, had a statistically significant higher HPVvaccination uptake. A negative association was showed between HPV-vaccination uptake and a municipal voting proportion for Party for Freedom and Forum for Democracy (PVV \& FvD), Christian Democratic Appeal (CDA) -only in the multivariable model-, Christian Union and Reformed political party (CU \& SGP) and Party for the Animals (PvdD) -only in the multivariable model- (Tables 2 and 3). This indicates that girls who 
lived in a municipality with a higher voting proportion for these parties, compared to the national mean, had a lower HPV-vaccination uptake.

Girls who lived in a municipality with a higher voting proportion for the populist parties with liberalconservative views PVV \& FvD had a significantly lower HPV-vaccination uptake in 2017, yet, in invitation years 2012 and 2014 either a positive or no statistically significant association between HPV-vaccination uptake and PVV \& FvD voting proportion was found (Table 3 and supplementary material 2 - model 1.1, 1.2, 2.7). A strong negative association between the HPV-vaccination uptake and the municipal voting proportions for the conservative Christian parties CU \& SGP was found for invitation years 2012, 2014 and 2017 (Table 2, Table 3, supplementary material 2 - model 1.2, 2.1,). This effect does not change over the invitation years (model 2.11).

\section{Discussion}

This study was performed to gain insight into the current relationship between social, economic and cultural determinants and the HPV-vaccination uptake of Dutch adolescent girls and whether the influence of these factors changed over time. Results showed that previous willingness to vaccinate (defined as MMRvaccination status), ethnicity, socioeconomic status of the postal code area, urbanization level of the municipality, road distance to vaccination location and municipal voting proportions in national elections were predictors for the HPV-vaccination uptake. Subgroups with a lower HPV-vaccination uptake in 2017 were in particular girls who have not received a MMR-vaccination (HPV-vaccine uptake $6.0 \%$ versus $48.9 \%$ when having received two MMR-vaccinations), who have one or two parents born in Morocco or Turkey (HPV-vaccine uptake 16.5-26.1\% versus $49.4 \%$ when having two parents born in the Netherlands), who live in an area with a lower socioeconomic status (HPV-vaccine uptake $36.9 \%$ versus $54.8 \%$ when socioeconomic status is high) and higher voting proportions in municipalities for Christian political parties (CU\&SGP) (HPV-vaccine uptake $41.1 \%$ versus $48.5 \%$ when voting proportions for Christian political parties are lower) and populist parties with liberal-conservative views (HPV-vaccine uptake $44.8 \%$ versus $48.1 \%$ when voting proportions for populist parties with liberalconservative views are lower). Besides some changes in political preferences of the population (association between HPV-vaccination uptake and higher voting proportions for populist parties with liberal-conservative views changed with an Adjusted OR (AOR) of 0.86 (95\% CI: $0.83-0.90)$ in 2017 versus 2012) and changes in the association between HPV-vaccination uptake and urbanization level (the difference in HPV-vaccination uptake between different levels of urbanization becomes smaller) we found no clear determinants which could possibly explain the decrease in the HPV-vaccination uptake.

Several groups in the Netherlands are known to have objections against vaccination in general. Among the orthodox Protestants, who live geographically clustered in the so-called Dutch Bible Belt, approximately $40 \%$ has not received childhood vaccinations [20]. In addition, people with affinity with an anthroposophical or natural lifestyle could also have a lower willingness to vaccinate $[21,22]$. In our multilevel multivariable logistic regression analysis, we used MMR-vaccination status to indicate people with a lower willingness to vaccinate in general. As expected, we found a significantly lower HPV-vaccination uptake among girls who had not received MMR-vaccinations in the past.

Regarding ethnicity, highest HPV-vaccination uptake was found among girls with both parents born in the Netherlands. Lowest uptake was in particular observed for girls with one or two parents born in Morocco or Turkey. This was also found in a study following the catch-up campaign in the Netherlands [7]. In a systematic review, belonging to minority racial or ethnic groups was also found as risk factors for low completion of HPV-vaccination series [23]. Parents of ethnic groups could be less proficient with the Dutch language and not responding to the invitation. Differences in culture and/ or religion could also explain this association [24, 25].

Girls living in areas with lower SES appeared to have lower HPV-vaccination uptake than girls living in areas with higher SES. This relation between SES and HPVvaccination uptake was also shown in a previous study in the Netherlands [7]. Underlying characteristics which play a role in SES are education level, having a payed job and the income of the household. Although vaccination was free of charge, a higher education level will help to better understand the usefulness of HPV-vaccination. In contrast, studies from England, Switzerland and the US showed that vaccination rates were lower in highincome families or in families with higher education [26-28]. Differences in healthcare systems and vaccination programs (i.e. school-based) between countries could lead to discrepancies in the association between SES and HPV-vaccination uptake.

In the most recent invitation year, 2017, a road distance to the vaccination location of more than five kilometers showed in the multilevel multivariable logistic regression model a very small but statistically significant association with a lower HPV-vaccination uptake. In contrast, no significant association was found between road distance to vaccination location and HPVvaccination uptake in 2012 and 2014. Another Dutch study showed that the average road distance was $5.7 \mathrm{~km}$ and was comparable between 2014 and 2017 [29]. People 
may have become more critical about travel distance nowadays. So, decreasing the road distance by expanding the number of vaccination locations, especially in rural areas, might help to increase the HPV-vaccination uptake but the magnitude of the effect is uncertain. In countries who have a school-based vaccination program (such as the UK and Australia), in which no additional traveling is necessary, the HPV-vaccination uptake is in general higher [30].

In 2012 and 2014, girls living in areas with higher urbanization levels had a lower HPV-vaccination uptake than girls living in areas with lower urbanization levels. However, in 2017, this association was not found. The Dutch study performed among girls eligible for the catch-up campaign in 2009 showed that unvaccinated girls lived in more urbanized areas [9]. In contrast, a study from Switzerland, showed that living in a rural municipality was associated with a lower uptake [28].

Regarding voting proportions in national elections, we found a lower HPV-vaccination uptake in girls living in municipalities with a higher voting proportions for the Christian political parties (CU\&SGP), compared to the national mean. The association between high political preference for Protestant Christian parties and low HPV-vaccination uptake was shown before in the Netherlands [7]. Apart from the objections to vaccination in general, various Christian groups have objections to HPV-vaccination in particular, because it concerns protection against a sexually transmitted disease $[7,9,31]$. A study in the US showed that adolescents from households with orthodox religious beliefs were almost 14 times less likely to get vaccinated [32]. In Switzerland, protestant religious groups were also associated with a lower uptake [28].

Also in 2017, a higher municipal voting proportion for populist parties with liberal-conservative views was found to be associated with a lower HPV-vaccination uptake. Previous database studies found that voters for Party for Freedom (PVV) and Forum for Democracy (FvD) may have less confidence in the government, media, and social institutions $[15,16]$. Also, some of the PVV \& FvD voters believe that the government hides information about the health risks of vaccines [16]. State-level voting patterns in the US, which may reflect population-level differences in cultural norms and social values, are also associated with uptake for adolescence vaccination [14].

In birth cohorts 2002 and 2003, i.e. who were vaccinated in 2015 and 2016, a sharp decrease in vaccination uptake was observed [5]. To study which determinants were associated with the decrease in the HPV-vaccination coverage it was investigated whether the influence of the various determinants changed over time. Results showed that the association with urbanization level was less clear in the invitation year 2017, compared with 2012 and 2014. Also, no association between the municipal voting rate for populist parties with liberal-conservative views was found in 2012 and 2014. However, in 2017 a high percentage of voters for populist parties with liberal-conservative views in the municipality was associated with a lower HPVvaccination uptake. This might be due to the lower confidence in the government, media and social institutions as mentioned before $[15,16]$. Besides the changes in political preferences of the population and changes in the association between HPV uptake and urbanization level we found no clear determinants associated with the decrease in the HPV-vaccination uptake. The decrease in HPVvaccination uptake may be more associated with a general decrease in trust in the vaccine and/or the fear of adverse events. Social media might have played a role in this.

Tailored strategies are critical in reaching groups with suboptimal vaccination uptake [33]. We were able to identify target groups that are currently associated with a lower HPV-vaccination rate in the Netherlands. Customized information and/or consultation might be useful to implement for low educated natives, girls with Moroccan or Turkish parents, girls with a Christian background and neighborhoods with a high proportion of voters for populist parties with liberal-conservative views to increase the HPV-vaccination uptake among these groups. Literature research also shows that reminders (before the vaccination moment), a no-show policy (such as a new invitation if one did not show up after the first invitation), customized information, feedback of the vaccination rate to professionals and making it easier to get the vaccinations, can lead to an increase the HPV-vaccination rate up to $10-20 \%$ [34]. Also other studies have been initiated in the Netherlands to reduce the inequalities in HPV vaccination uptake [35, 36].

Besides the strength that individual data was used on vaccination status to determine the HPV-vaccination uptake, this study has also some limitations. Data on social, economic, cultural and political determinants were not collected for the purpose of this study and only available on postal code level or municipality level. Therefore, associations on these aggregation levels represent the group of individuals within a given area and might not directly apply to an individual. For example, it concerns the voting behavior of adults in the municipality, while these girls were not yet allowed to vote themselves. On the other hand, the decision about vaccination is also mostly made by the parents of the girls. Furthermore, for some determinants data was not available for the specific years included in this study. In this case the most recent data was used. Proportions for the political parties in national elections were only available for 2012 and 2017. For road distance, only data was available for 2014 and 2017. Therefore, the results for 2012 and 2014 should be interpreted with caution. Also, we used home addresses obtained in 2018. Girls might have been moved in 
the years before, but we think that these movements outweigh each other and therefore had a very small effect on the analyses. Besides that, some variables contained a large number of missings. Especially for ethnicity, which counted low numbers for some categories in all cohorts, especially in 2012 and 2014. This limits the comparability of these variables over time. Besides the investigated determinants, there are other determinants that are possibly associated with the HPV-vaccination uptake. For example, schooleducation or being the oldest girls in the family (i.e. the first who is eligible for HPV-vaccination). Unfortunately, no information on these or other potential determinants was available in the databases.

\section{Conclusions}

In this study we identified current social, economic and cultural determinants that are associated with HPVvaccination uptake for public health relevance. Customized information and/or consultation should be prepared for identified target groups that are associated with a lower HPV-vaccination rate. We found no clear determinants which explain the decrease in the HPV-vaccination uptake. The vaccination coverage recently increased again in the Netherlands [37], probably fostered by the Meningococcal ACWY vaccination campaign for adolescents. This shows that it is possible to increase the vaccination coverage and protect more girls against cervical cancer. This positive message might help to increase the HPVvaccination coverage in the Netherlands further.

\begin{abstract}
Abbreviations
50+: 50PLUS (party that stands up especially for the interests of people aged 50 and over); 95\%Cl: 95\% confidence intervals; AOR: adjusted odds ratios; CBS: Statistics Netherlands; CDA: Christian Democratic Appeal (Christian-inspired party at the center of the political spectrum); COR: crude odds ratios; CU: Christian Union (Christian party, with progressive positions in the social and ecological field and conservative positions on ethical issues).; D66 : Democrats 66 (reformist social-liberal party); DENK: Denk (movement for migrants and a "tolerant and solidary society"); FvD: Forum for Democracy (conservative, right-wing populist Eurosceptic political party); $\mathrm{GL}$ : Green party (progressive party which attaches great importance to sustainability); HPV: human papillomavirus; MHS: Municipal Health Services; MMR: mumps, measles and rubella; NIP: national immunization program; OR: odds ratios; PvdA: Labor Party (progressive, social-democratic party); PvdD: The Party for the Animals (testimonial party with as main goals animal rights and animal welfare); PW: Party for Freedom (populist party with both conservative, liberal "right" and "left" views); SCP: The Netherlands Institute for Social Research; SGP: Reformed Political Party (conservative Christian (Reformed) party that wants to conduct politics strictly according to Biblical standards).; SP: Socialist Party (socialist, Eurosceptic party which has a strong local, action-oriented basis); WD: People's Party for Freedom and Democracy (right-wing liberal party with more progressive positions in ethical matters).
\end{abstract}

\section{Supplementary Information}

The online version contains supplementary material available at https://doi. org/10.1186/s12889-021-11897-0.

Additional file 1.

Additional file 2 .

\section{Acknowledgements}

Thanks to Petra Oomen to provide the data form Praeventis.

\section{Authors' contributions}

AdM analyzed and interpreted the data. TSK prepared a part of the dataset and also interpreted the data. AdM and TSK both contributed in writing and revising the manuscript. AvL, HdM and WR substantially contributed in the whole study process and they revised the manuscript several times. RA contributed to the statistical analyses. All authors read and approved the final manuscript.

\section{Funding}

This research study was (partially) supported by the research fund of the Dutch National Institute for Public Health and the Environment (RIVM), the Netherlands, for local Public Health Services.

\section{Availability of data and materials}

All data generated or analysed during this study are included in this published article and its supplementary information files.

\section{Declarations}

Ethics approval and consent to participate

The study was approved by the research ethics committee of the Radboud University Nijmegen Medical Centre, Nijmegen, the Netherlands; CMO number 2018/4744. The Præventis data were provided anonymized to the researchers after approval by the registration commission. Therefore the need for consent of the participants was waived off by the research ethics committee of the Radboud University Nijmegen Medical Centre, Nijmegen, the Netherlands. This study was conducted according to the principles of the most recent World Medical Association Declaration of Helsinki and in accordance with the Medical Research Involving Human Subjects Act (WMO).

\section{Consent for publication}

NA

\section{Competing interests}

The authors declare that they have no competing interests.

\section{Author details}

'Department of Infectious Disease Control, Public Health Service Gelderland-Zuid, Nijmegen, The Netherlands. ${ }^{2}$ Radboud University Nijmegen Medical Centre, Department of Primary and Community Care \& IQ Health care, Nijmegen, The Netherlands. ${ }^{3}$ Radboud University Medical Centre, Radboud Institute for Health Sciences, IQ healthcare, Nijmegen, The Netherlands. ${ }^{4}$ Department National Immunization Program, Center of Epidemiology and Surveillance of Infectious Diseases, National Institute for Public Health and the Environment, PO box 1, 3720, BA, Bilthoven, The Netherlands. ${ }^{5} \mathrm{GGD}$ GHOR Nederland, Utrecht, The Netherlands.

Received: 16 February 2021 Accepted: 29 September 2021

Published online: 12 October 2021

\section{References}

1. Clifford G, Franceschi S, Diaz M, Munoz N, Villa LL. Chapter 3: HPV typedistribution in women with and without cervical neoplastic diseases. Vaccine. 2006;24(Suppl 3):S3/26-34.

2. Integraal kankercentrum Nederland (IKNL). Nederlandse Kankerregistratie Utrecht 2017 [Available from: http://www.cijfersoverkanker.nl/.

3. Integraal kankercentrum Nederland (IKNL). Incidentie, sterfte en overleving baarmoederhalskanker. 17 September 2018. Available from: https://www. iknl.nl/nieuws/2018/incidentie,-sterfte-en-overleving-baarmoederhalska.

4. van Lier E, Oomen P, Giesbers H, Drijfhout I, de Hoogh P, de Melker H. Immunization coverage National Immunization Programme in the Netherlands: Year of report 2011. Bilthoven: RIVM; 2011. RIVM report 210021014.

5. Van Lier EA, Oomen PJ, Giesbers H, van Vliet JA, Drijfhout IH, ZonnenbergHoff IF, et al. Immunisation coverage and annual report National Immunisation Programme in the Netherlands 2018. Bilthoven: RIVM; 2019. RIVM report 2019-0015. 
6. Volksgezondheidenzorg.info [20-02-2020]. Available from: https:/wwww. volksgezondheidenzorg.info/onderwerp/vaccinaties/regionaal-internationaa I/adolescenten\#node-hpv-vaccinaties-gemeente.

7. Rondy M, van Lier A, van de Kassteele J, Rust L, de Melker H. Determinants for HPV vaccine uptake in the Netherlands: a multilevel study. Vaccine. 2010; 28(9):2070-5. https://doi.org/10.1016/.jvaccine.2009.12.042.

8. van Keulen HM, Otten W, Ruiter RA, Fekkes M, van Steenbergen J, Dusseldorp E, et al. Determinants of HPV vaccination intentions among Dutch girls and their mothers: a cross-sectional study. BMC Public Health. 2013;13:111.

9. Mollers M, Lubbers K, Spoelstra SK, Weijmar-Schultz WC, Daemen T, Westra $T A$, et al. Equity in human papilloma virus vaccination uptake?: sexual behaviour, knowledge and demographics in a cross-sectional study in (un) vaccinated girls in the Netherlands. BMC Public Health. 2014;14:288.

10. Ruijs WL, Hautvast JL, van der Velden K, de Vos S, Knippenberg H, Hulscher ME. Religious subgroups influencing vaccination coverage in the Dutch bible belt: an ecological study. BMC Public Health. 2011;11:102.

11. Marlow LA, Waller J, Wardle J. Trust and experience as predictors of HPV vaccine acceptance. Hum Vaccin. 2007 Sep-Oct;3(5):171-5. https://doi.org/1 0.4161/hv.3.5.4310

12. Harrington N, Chen Y, O'Reilly AM, Fang CY. The role of trust in HPV vaccine uptake among racial and ethnic minorities in the United States: a narrative review. AIMS Public Health. 2021 Apr 6;8(2):352-68.

13. Gefenaite G, Smit M, Nijman HW, Tami A, Drijfhout IH, Pascal A, et al. Comparatively low attendance during human papillomavirus catch-up vaccination among teenage girls in the Netherlands: insights from a behavioral survey among parents. BMC Public Health. 2012 Jul 2;12:498.

14. Bernstein S, North A, Schwartz J, Niccolai LM. State-level voting patterns and adolescent vaccination coverage in the United States, 2014. Am J Public Health. 2016;106(10):1879-81. https://doi.org/10.2105/AJPH.2016.303381.

15. Centraal Bureau voor de Statistiek (CBS). Het profiel van het electoraat in 2012 Den Haag2017 [Available from: https://www.cbs.nl/nl-nl/achtergrond/2 017/08/het-profiel-van-het-electoraat-in-2012.

16. Wendelmoet Boersema, Romana Abels. Onderzoek Kieskompas: Complotten? Kiezers van FvD, PW en 50Plus zweren erbij 2019 [07-122019]. Available from: https://www.trouw.nl/binnenland/complotten-kiezersvan-fvd-pvv-en-50plus-zweren-erbij b2138a0d/?utm_campaign=shared_ea rned\&utm_medium=social\&utm_source=email.

17. Parlement.com. Partijen in Tweede en Eerste Kamer. Available from: https:// www.parlement.com/id/vh8Inhrpfxut/partijen_in_tweede_en_eerste_kamer.

18. Field A. Discovering statistics using IBM SPSS. 5th ed. Thousand Oaks, CA: Sage Publications Ltd; 2017.

19. Steyerberg EW, Vergouwe Y. Towards better clinical prediction models: seven steps for development and an ABCD for validation. Eur Heart J. 2014; 35(29):1925-31. https://doi.org/10.1093/eurheartj/ehu207.

20. Spaan DH, Ruijs WLM, Hautvast JLA, Tostmann A. Increase in vaccination coverage between subsequent generations of orthodox Protestants in the Netherlands. Eur J Pub Health. 2017;27(3):524-30. https://doi.org/10.1093/ eurpub/ckw248.

21. Klomp JH, van Lier A, Ruijs WL. Vaccination coverage for measles, mumps and rubella in anthroposophical schools in Gelderland, the Netherlands. Eur J Pub Health. 2015;25(3):501-5.

22. Harmsen IA, Ruiter RA, Paulussen TG, Mollema L, Kok G, de Melker HE. Factors that influence vaccination decision-making by parents who visit an anthroposophical child welfare center: a focus group study. Adv Prev Med. 2012;2012:175694.

23. Gallagher KE, Kadokura E, Eckert LO, Miyake S, Mounier-Jack S, Aldea M, et al. Factors influencing completion of multi-dose vaccine schedules in adolescents: a systematic review. BMC Public Health. 2016;16:172.

24. Zouheir Y, Daouam S, Hamdi S, Alaoui A, Fechtali T. Knowledge of human papillomavirus and acceptability to vaccinate in adolescents and young adults of the Moroccan population. J Pediatr Adolesc Gynecol. 2016 Jun; 29(3):292-8.

25. Mouallif M, Bowyer HL, Festali S, Albert A, Filali-Zegzouti Y, Guenin S, et al. Cervical cancer and HPV: awareness and vaccine acceptability among parents in Morocco. Vaccine. 2014 Jan 9;32(3):409-16. https://doi.org/10.101 6/j.vaccine.2013.10.069.

26. Jean S 3rd, Elshafei M, Buttenheim A. Social determinants of communitylevel human papillomavirus vaccination coverage in a school-based vaccination programme. Sex Transm Infect. 2018;94(4):248-53. https://doi. org/10.1136/sextrans-2017-053357.
27. Vu M, Luu M, Haardorfer R, Berg CJ, Escoffery C, Bednarczyk RA. A multilevel analysis of factors influencing the inaccuracy of parental reports of adolescent HPV vaccination status. Vaccine. 2019;37(6):869-76. https://doi. org/10.1016/j.vaccine.2018.12.032.

28. Riesen M, Konstantinoudis G, Lang P, Low N, Hatz C, Maeusezahl M, et al. Exploring variation in human papillomavirus vaccination uptake in Switzerland: a multilevel spatial analysis of a national vaccination coverage survey. BMJ Open. 2018;8(5):e021006. https://doi.org/10.1136/bmjopen-2017-021006.

29. Quee FA, Mollema L, van Vliet JA, de Melker HE, van Lier EA. No link between organisational changes in youth healthcare services and the trend in vaccination levels from 2013 to 2017 Bilhoven: RIVM; 2018. Report No.: RIVM briefrapport 2018-0111.

30. Hofstetter AM, Rosenthal SL. Factors impacting HPV vaccination: lessons for health care professionals. Expert Rev Vaccines. 2014 Aug;13(8):1013-26. https://doi.org/10.1586/14760584.2014.933076.

31. Shelton RC, Snavely AC, De Jesus M, Othus MD, Allen JD. HPV vaccine decision-making and acceptance: does religion play a role? J Relig Health. 2013;52(4):1120-30. https://doi.org/10.1007/s10943-011-9553-x.

32. Sriram $S$, Ranganathan $R$. Why human papilloma virus vaccination coverage is low among adolescents in the US? A study of barriers for vaccination uptake. Journal of family medicine and primary care. 2019;8(3):866-70. https://doi.org/10.4103/ffmpc.jfmpc_107_19.

33. Dube E, Leask J, Wolff B, Hickler B, Balaban V, Hosein E, et al. The WHO tailoring immunization Programmes (TIP) approach: review of implementation to date. Vaccine. 2018;36(11):1509-15. https://doi.org/10.101 6/j.vaccine.2017.12.012

34. Mollema L, Antonise-Kamp L, van Vliet J, de Melker H. Organisatorische en communicatieve interventies die de opkomst voor HPV-vaccinatie kunnen verhogen [In Dutch]. Tijdschr Jeugdgezondheidsz. 2019;51:101-5.

35. Den Haag. Grote vaccinatie-verschillen tussen kinderen met en zonder migratieachtergrond. Available from: https://www.denhaag.nl/nl/in-de-stad/ nieuws/pers/grote-vaccinatie-verschillen-tussen-kinderen-met-en-zondermigratieachtergrond.htm [01-06-2021].

36. EuroHealthNet. New initiative launched to improve access to MMR and HPV vaccines in underserved populations. Press release 01-06-2021. Available from: https://eurohealthnet.eu/media/news-releases/new-initiative-launchedimprove-access-mmr-and-hpv-vaccines-underserved [02-06-2021].

37. van Lier EA, Kamp L, Oomen PJ, Giesbers H, van Vliet JA, Drijfhout IH, et al. Immunisation coverage and annual report National Immunisation Programme in the Netherlands 2019. Bilthoven: RIVM; 2020. RIVM report 2020-0011.

\section{Publisher's Note}

Springer Nature remains neutral with regard to jurisdictional claims in published maps and institutional affiliations.

Ready to submit your research? Choose BMC and benefit from:

- fast, convenient online submission

- thorough peer review by experienced researchers in your field

- rapid publication on acceptance

- support for research data, including large and complex data types

- gold Open Access which fosters wider collaboration and increased citations

- maximum visibility for your research: over $100 \mathrm{M}$ website views per year

At BMC, research is always in progress.

Learn more biomedcentral.com/submissions 